\title{
Secuestro pulmonar. Serie clínica de 16 pacientes
}

\author{
GUILLERMO ZEPEDA F.***, RODRIGO BOZZO H.***, MARGARITA ALDUNATE R.****, \\ LUIS VELOZO P.***** y CAROLINA GVIRTZMAN K.*
}

\section{Pulmonary sequestration. Clinical report of 16 patients}

Objective: To describe our experience with sixteen children treated for pulmonary sequestration at the Children's Hospital Roberto del Rio, between 2000 and 2012. Method: We reviewed retrospectively the clinical charts of all those patients up to 15 years old, who were treated for pathologically proven pulmonary sequestration in our hospital, between 2000 and 2012. Results: Sixteen patients were recorded, 8 boys and 8 girls that were diagnosed and/or treated between the newborn period and 14 years old. Nine of them had extralobar sequestration and 7 intralobar. The diagnosis was suspected for persisting pneumonia in 44\%, neonatal respiratory distress in $25 \%$, prenatal ultrasound in 19\%, and recurrent pneumonia in 12\%. Seventy-five percent of sequestrations were located in the left lower lobe, $19 \%$ in the right lower lobe and 6\% in the right upper lobe and all of them were operated via thoracotomy. Fifty-six percent underwent surgery before one year of age (31.5\% before 2 weeks of life) and $69 \%$ of patients did well postoperatively. Postoperative atelectasis were detected in $12.5 \%$, bronchopleural fistula in $12.5 \%$ and residual pneumothorax in $6 \%$. The average hospital stay was 9 days and no mortality was registered. Conclusions: Our experience is similar to the previously reported series and emphasizes the importance of early diagnosis in the treatment of pulmonary sequestration in children.

Key words: Pulmonary sequestration, extralobar, intralobar, brochiopulmonary malformations.

\section{Resumen}

Objetivo: Describir los hallazgos clínicos de pacientes tratados por secuestro pulmonar en el Hospital de Niños Roberto del Río, entre los años 2000 y 2012. Métodos: Estudio descriptivo retrospectivo. Se revisaron las fichas clínicas de todos los pacientes atendidos en el Hospital entre el año 2000 y 2012 con diagnóstico de secuestro pulmonar confirmado por anatomía patológica. Resultados: Se registraron 16 pacientes, 8 niños y 8 niñas. Del total 9 fueron secuestros extralobares y 7 intralobares. La sospecha diagnóstica fue por imagen de neumonía persistente en el 44\% de los casos, distrés respiratorio neonatal en el 25\%, ecografía antenatal en el 19\% y neumonía repetida en el $12 \%$. El 75\% de los secuestros estaba ubicado en el lóbulo inferior izquierdo, el 19\% en lóbulo inferior derecho y el $6 \%$ en lóbulo superior derecho. La técnica quirúrgica de elección fue la toracotomía abierta. El 56\% de los pacientes se operó antes del año de vida (31,5\% antes de las 2 semanas de vida). La evolución postoperatoria fue buena en el $69 \%$ de los casos, mientras que un 12,5\% presentó atelectasia, 12,5\% fistula broncopleural y 6\% neumotórax residual. La mediana de estadía hospitalaria fue de 9 días. No se registró mortalidad. Conclusiones: Se presenta la experiencia de esta patología en el Hospital Roberto del Río enfatizándose la importancia de la sospecha diagnóstica.

Palabras clave: Secuestro pulmonar, secuestro extralobar, secuestro intralobar, malformaciones pulmonares.

\footnotetext{
* Programa de Enfermedades Respiratorias Pediátricas. Departamento de Pediatría y Cirugía Infantil, Campus Norte de la Facultad de Medicina Universidad de Chile. Hospital Roberto del Río.

** $\quad$ Magister en Educación en Ciencias de la Salud. Facultad de Medicina Universidad de Chile.

*** Broncopulmonar infantil. Hospital Roberto del Río.

**** Cirujana infantil. Departamento de Pediatría y Cirugía Infantil, Campus Norte de la Facultad de Medicina Universidad de Chile. Hospital Roberto del Río.

*****Anatomopatólogo. Hospital Roberto del Río.
} 


\section{Introducción}

El secuestro pulmonar se ha definido como un segmento de pulmón que no tiene comunicación identificable con el árbol traqueobronquial normal y que recibe irrigación arterial sistémica desde una o más arterias anómalas ${ }^{1}$.

Se clasifica en dos tipos sobre la base de su cubierta pleural y su patogenia. El secuestro extralobar es una masa de tejido pulmonar que tiene una cubierta pleural distinta manteniendo la separación anatómica completa respecto del tejido pulmonar normal adyacente y es una malformación congénita, equivalente a un tejido pulmonar ectópico ${ }^{2}$. En cambio, el secuestro intralobar es una masa de parénquima pulmonar independiente, que es contigua al tejido pulmonar normal ${ }^{1,2}$. Según Stocker ${ }^{3}$, el secuestro intralobar, es el resultado de un proceso inflamatorio adquirido que aísla un segmento pulmonar del árbol bronquial, se organiza y adquiere circulación arterial propia desde vasos que sufren hipertrofia, ubicados en los ligamentos pulmonares ${ }^{3}$.

La histopatología de los secuestros extralobares corresponde a tejido pulmonar normal mezclado con tejido con alteraciones malformativas similares a una malformación adenomatoídea quística tipo 2 de la vía aérea, más una arteria sistémica ${ }^{2,3}$. En cambio, en el intralobar hay un daño pulmonar crónico, con inflamación mixta exudativa, linfoplasmocitaria, xantomatosa, presencia de quistes, neumatoceles o bronquiectasias, además una arteria nutricia sistémica de calibre bastante grueso ${ }^{2,3}$.

La sintomatología causada por los secuestros es diferente según el tipo de secuestro, ubicación, tamaño, asociación con otras malformaciones, complicaciones, etc ${ }^{4-14}$.

En Chile no existen series clínicas recientes que describan esta patología, la última serie clínica que encontramos publicada a nivel nacional data del año 1982 en que se reportaron 6 casos de secuestros intralobares ${ }^{15}$.

Debido a lo anterior, el objetivo de este estudio es presentar la experiencia acumulada tanto de secuestros extralobares como intralobares en el Hospital Roberto del Río en los últimos años de manera de contribuir al mejor entendimiento de esta patología.

\section{Metodología}

Estudio de tipo retrospectivo en el cual se realizó una búsqueda en la base de datos del Servicio de Anatomía Patológica del Hospital Roberto del Río de los pacientes operados de secuestro pulmonar registrados desde enero de 2000 hasta el segundo semestre del año 2012. Luego se procedió a extraer de las fichas clínicas de los pacientes los datos demográficos, clínicos, radiológicos y quirúrgicos y se llevaron a planilla de cálculo Excel para el análisis respectivo. Los datos son expresados como valores absolutos, porcentajes o mediana.

\section{Resultados}

Se registraron 16 pacientes en el período 8 de ellos del género femenino. Los tipos de secuestros fueron 9 extralobares y 7 intralobares. La distribución por edad indicó una edad de diagnóstico y/u operación en que predominaron los menores de 1 año con 9 pacientes, de los cuales 5 fueron recién nacidos (Tabla 1 y Figura 1 ).

La ubicación según el lóbulo comprometido indicó un claro predominio de los lóbulos inferiores con un $94 \%$ del total, especialmente del lóbulo inferior izquierdo (LII) con un $75 \%$ (Tabla 2).

La ubicación de los secuestros extralobares fue bajo el LII en 6 casos, bajo el lóbulo inferior derecho en 2 casos y bajo el lóbulo superior derecho en un caso. En relación a los secuestros intralobares 6 se ubicaron en el LII y uno en el lóbulo inferior derecho (Tabla 3). No se registraron casos de ubicación ectópica de secuestros.

El diagnóstico imagenológico se logró con tomografía computada (TC) en 9 pacientes, Angio-TC en 4 pacientes, angiografía convencional en 2 pacientes y en un paciente se sospechó el diagnóstico con radiografía de tórax (operado de hernia diafragmática) (Figura 2).

La forma de presentación en los secuestros extralobares indicó un predominio del diagnóstico prenatal y el síndrome de distrés respiratorio neonatal y en los secuestros intralobares predominó la neumonía repetida y las imágenes radiológicas persistentes (Tabla 4).

En relación a las comorbilidades se objetivaron en 8 pacientes, 5 con secuestro extralobar y 3 con secuestro intralobar. Las comorbilidades fueron: hernia diafragmática, dextrocardia, síndrome de Prune Belly, pubertad precoz, comunicación interauricular, sin objetivar un claro predominio de alguna en particular (Tabla 5). No hubo otras malfomaciones pulmonares asociadas.

El tipo de cirugía dependió del tipo de secuestro. De esta manera en los pacientes con secuestros extralobares se realizó secuestrectomía y en los pacientes con secuestros intralobares se realizó lobectomía.

El método operatorio más utilizado fue la toracotomía abierta en 13 pacientes $(81 \%)$, en 
2 pacientes (13\%) se inició videotoracoscopía (VATS) y se tuvo que reconvertir a cirugía abierta y en un paciente $(6 \%)$ se realizó exitosamente la VATS de su secuestro extralobar.

Los pacientes evolucionaron en general satisfactoriamente. De esta manera, no se objetivó complicaciones en $69 \%$, siendo las fístulas broncopleurales las únicas complicaciones mayores (Tabla 6). No se registró mortalidad.

La mediana de estadía hospitalaria post-cirugía correspondió a 9 días con un rango de 3 a 46 días, correspondiendo este último valor a un paciente que evolucionó con una fístula broncopleural. Se excluyó a los recién nacidos (en total 5) de este análisis debido a la dificultad para realizar un análisis sólo del acto operatorio en virtud de patologías neonatales médicas. Sin embargo, los que no tenían otras patologías fueron dados de alta al segundo o tercer día postoperatorio.

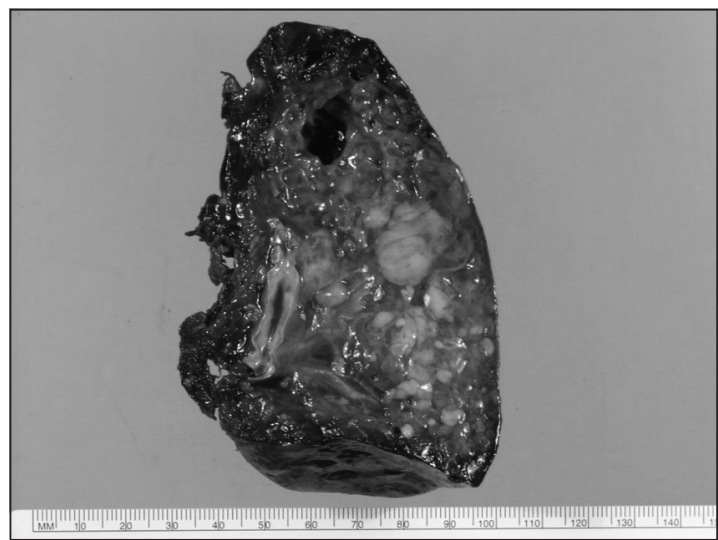

Figura 1. Secuestro intralobar infectado en lóbulo inferior izquierdo de un paciente operado a los 10 meses de edad, con sospecha diagnóstica de imagen persistente radiológica.

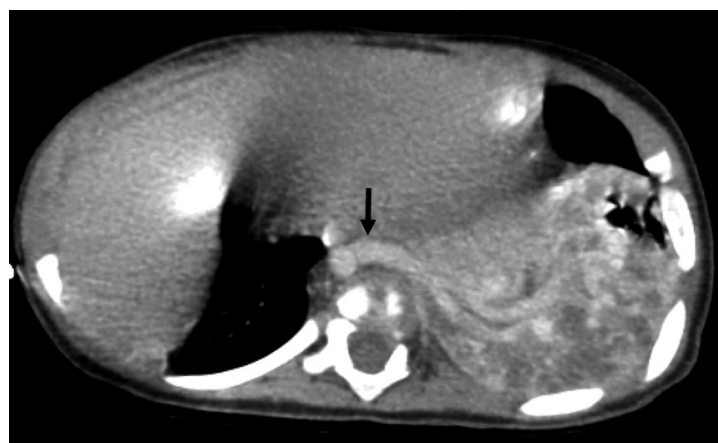

Figura 2. AngioTC de paciente con diagnóstico de secuestro intralobar del lóbulo inferior izquierdo, de 8 meses de edad al momento de obtener esta imagen (es el mismo paciente de la Figura 1). Nótese el tamaño de la irrigación arterial aberrante proveniente de la aorta torácica descendente (indicado por la flecha).
Tabla 1. Datos demográficos de 16 pacientes operados de secuestro pulmonar

\begin{tabular}{|c|c|}
\hline Sexo: Femenino/Masculino (n/n) & $8 / 8$ \\
\hline \multicolumn{2}{|c|}{ Edad al efectuar la intervención quirúrgica } \\
\hline Menores de 1 año n (\%) & $9(56)$ \\
\hline Menores de 1 mes $\mathrm{n}$ & $\begin{array}{l}5\left(2^{\circ}, 6^{\circ}, 8^{\circ}, 13^{\circ} \text { y }\right. \\
\left.14^{\circ} \text { día de vida }\right)\end{array}$ \\
\hline 1 a 5 años n $(\%)$ & $2(13)$ \\
\hline 6 a 10 años n $(\%)$ & $1(6)$ \\
\hline 10 a 14 años n $(\%)$ & $4(25)$ \\
\hline $\begin{array}{l}\text { Tipo de secuestro pulmonar } \\
\text { Extralobar/Intralobar n (\%) }\end{array}$ & $9 / 7(56 / 44)$ \\
\hline
\end{tabular}

Tabla 2. Ubicación de secuestros pulmonares en 16 pacientes según el lóbulo afectado

\begin{tabular}{|lccrr|}
\hline Localización & Derecho & Izquierdo & \multicolumn{2}{c|}{ Total } \\
& & & n & \% \\
\hline Lóbulo inferior & 3 & 12 & 15 & 94 \\
\hline Lóbulo superior & 1 & 0 & 1 & 6 \\
\hline Lóbulo medio & 0 & 0 & \multicolumn{2}{c}{0} \\
\hline
\end{tabular}

Tabla 3. Ubicación de secuestros pulmonares extralobares e intralobares según lóbulo afectado en 16 pacientes

\begin{tabular}{|lccccc|}
\hline & Extralobar & Intralobar & \multicolumn{3}{c|}{ Total } \\
& & \multicolumn{2}{c}{ n } & \% \\
LSD & 1 & 0 & 1 & 6 \\
LMD & 0 & 0 & \multicolumn{2}{c}{0} & \\
LID & 2 & 1 & 3 & 19 \\
LSI & 0 & 0 & & 0 & \\
LII & 6 & 6 & 12 & 75 \\
\hline
\end{tabular}

LSD: lóbulo superior derecho. LMD: lóbulo medio derecho. LID: lóbulo inferior derecho. LSI: lóbulo superior izquierdo. LII: lóbulo inferior izquierdo.

Tabla 4. Forma de presentación del secuestro pulmonar en 16 pacientes

\begin{tabular}{|lcccc|}
\hline & $\begin{array}{c}\text { Extra- } \\
\text { lobar }\end{array}$ & $\begin{array}{c}\text { Intra- } \\
\text { lobar }\end{array}$ & \multicolumn{2}{c|}{ Total } \\
\hline Diagnóstico prenatal & 3 & 0 & 3 & 19 \\
$\begin{array}{l}\text { Diagnóstico neonatal } \\
\text { SDR }\end{array}$ & 4 & 0 & 4 & 25 \\
\hline $\begin{array}{l}\text { Imagen persistente } \\
\text { Neumonía repetida }\end{array}$ & 2 & 5 & 7 & 44 \\
\hline
\end{tabular}

SDR: síndrome de distrés respiratorio. 
Tabla 5. Comorbilidades según tipo de secuestro pulmonar en 16 pacientes

\begin{tabular}{|lcc|}
\hline & $\begin{array}{c}\text { Extralobar } \\
\text { (n) }\end{array}$ & $\begin{array}{c}\text { Intralobar } \\
\text { (n) }\end{array}$ \\
\hline Hernia diafragmática & 2 & 0 \\
Dextrocardia & 1 & 0 \\
Fisura palatina & 0 & 1 \\
Quiste de duplicación & 1 & 0 \\
CIA & 1 & 0 \\
Pubertad precoz & 0 & 1 \\
Prune Belly & 0 & 1 \\
Total & 5 & 3 \\
\hline
\end{tabular}

CIA: comunicación interauricular.

Tabla 6. Evolución de 16 pacientes operados de secuestro pulmonar

\begin{tabular}{|lcc|}
\hline Evolución & $\mathbf{n}$ & $\mathbf{\%}$ \\
\hline Satisfactoria & 11 & 69 \\
\hline Atelectasia & 2 & 12,5 \\
\hline Fístula broncopleural & 2 & 12,5 \\
\hline Neumotórax residual & 1 & 6 \\
\hline
\end{tabular}

\section{Discusión}

Las malformaciones congénitas torácicas son un grupo heterogéneo de raros desórdenes que pueden involucrar la vía aérea o el parénquima pulmonar ${ }^{16}$. Esta denominación ha sido propuesta recientemente en un artículo avalado por la European Respiratory Society y su clasificación es la siguiente: malformación adenomatoidea quística congénita, hernia diafragmática congénita, fístula tráqueo-esofágica, secuestro pulmonar, quistes, tumores, atresia bronquial con degeneración distal, enfisema lobar congénito, pulmón pequeño congénitamente, agenesia pulmonar y anormalidades vasculares ${ }^{16}$.

Los secuestros pulmonares son una de las causas importantes de malformaciones congénitas torácicas que habitualmente se detectan en la infancia, pero que ocasionalmente puede diagnosticarse en la edad adulta pudiendo ocasionar diversas complicaciones $5,7,17,18$.

La serie clínica presentada aunque pequeña nos indica diversos hechos relevantes. De esta manera, no existió en nuestros pacientes predominio de género en el total de secuestros ni en el tipo de éstos, lo cual se contrasta con lo relatado por $\mathrm{Wei}^{5}$, en que indica una relación de 1,58:1 a favor de los hombres, siendo ésta la serie publicada con mayor número de casos registrados a la fecha ${ }^{5}$.

Destacamos en nuestra serie la precocidad en el diagnóstico, correspondiendo los diagnósticos prenatales al 19\% del total de casos (todos secuestros extralobares) lo cual traduce la acuciosidad en la realización de las ecografías obstétricas. Este importante punto, tal como lo relatan diferentes autores, es crucial para un manejo oportuno del secuestro como de las potenciales complicaciones tanto intrauterinas como extrauterinas, debiéndose complementar la ecografía con una resonancia magnética fetal en algunos casos justificados ${ }^{19,20}$.

Nuestros pacientes con diagnóstico prenatal y/o neonatal precoz fueron operados en forma temprana como se ha sugerido en la literatura con la finalidad de evitar las complicaciones propias de esta patología ${ }^{21}$.

En relación a los lóbulos comprometidos, nuestros resultados indican un claro predominio de los lóbulos inferiores con un $94 \%$ tal como se ha señalado previamente en la literatura en las principales casuísticas. $\mathrm{Wei}^{5}$ describe un $97 \%$ de afección de los lóbulos inferiores y Savic ${ }^{4}$ porcentajes de $97,75 \%$ para los secuestros intralobares y de $77,4 \%$ para los secuestros extralobares (estos últimos ubicados debajo de los lóbulos inferiores $)^{4,5}$.

En la casuística descrita, logramos objetivar compromiso del LII en un $75 \%$ de los casos, lo cual concuerda con los datos entregados por $\mathrm{Wei}^{5}$ en que relata un $71,5 \%$ de compromiso del $\mathrm{LII}^{5} \mathrm{y}$ de Savic ${ }^{4}$ en que describe la afección del LII en un $72 \%$ de los secuestros extralobares (debajo del LII) y de $57 \%$ de los secuestros intralobares ${ }^{4,5}$. En relación a los segmentos comprometidos destaca el segmento basal posterior de ambos lóbulos inferiores como el de mayor compromiso con un $87 \%$ del total de los secuestros ${ }^{5}$ no pudiendo confrontarse este valor con nuestros datos.

En nuestra serie no hubo secuestros ectópicos a diferencia de lo relatado en la literatura en que se informan ubicaciones en regiones de mediastino, paracardíaca, infradiafragmática y en la cavidad abdominal ${ }^{4}$.

La forma de presentación dependió del tipo de secuestro y de la ubicación, tal como se ha señalado en la literatura ${ }^{1,4,5}$. En este punto es importante la sospecha diagnóstica cuando existen lesiones pulmonares que no mejoran después de una consolidación parenquimatosa considerada como neumonía, ya que los secuestros pueden causar neumonía recurrente en una misma área del pulmón ${ }^{6}$. Así, en nuestra serie se presentaron 
2 pacientes derivados de distintas regiones de Chile con diagnóstico de neumonía repetida en los que probablemente un control radiológico posterior al tratamiento antibiótico hubiera contribuido a lograr una sospecha diagnóstica más certera.

Cabe destacar que en nuestros pacientes con secuestro intralobar se objetivaron casos de infección tal como se ha descrito previamente ${ }^{5,7}$.

En la literatura se han referido otras formas de presentación: simulación de otra patología como enfisema lobar congénito ${ }^{8}$, asociación con otras patologías como malformaciones congénitas de la vía aérea y quistes broncogénicos ${ }^{9} \mathrm{o}$ compromiso bilateral ${ }^{5,10}$; ninguna de estas fueron encontradas en el presente estudio. Tampoco se registraron complicaciones de los secuestros extralobares como torsión del pedúnculo arterial (presentación como dolor torácico) $)^{11}$, o infarto del secuestro ${ }^{12,13}$ con hemotórax secundario ${ }^{14}$.

La imagenología es fundamental para lograr un diagnóstico preoperatorio óptimo especialmente en lo que concierne a la ubicación de los vasos sanguíneos aberrantes. Nuestros pacientes fueron estudiados predominantemente con TC pulmonar debido a que sólo en los últimos años se ha tenido una mayor disponibilidad para realizar AngioTC en nuestro centro. Este punto concuerda con lo señalado en la literatura en el sentido de que los exámenes con mejor rendimiento serían el Angio-TC o la Angio-RM dependiendo de la disponibilidad de cada centro ${ }^{5,22,23}$, los que pueden incluir imágenes tridimensionales que tendrían mejor rendimiento para detectar los vasos sanguíneos ${ }^{22}$.

Las comorbilidades asociadas fueron variadas y no se objetivó un predominio en relación al tipo de secuestro como se ha señalado en la literatura donde predominan las comorbilidades especialmente en el secuestro extralobar ${ }^{1}$, ni tampoco se registró una mayor cantidad de alteraciones a nivel diafragmático como se ha relatado previamente ${ }^{1,4,5,24}$.

El tipo de cirugía varió dependiendo de si se trataba de un secuestro extralobar o intralobar realizándose secuestrectomía o lobectomía respectivamente lo cual concuerda con lo descrito previamente ${ }^{1,5,25}$. El método utilizado preferido fue la vía abierta debido a la factibilidad de su uso, a pesar de que la videotoracoscopía es una posibilidad cierta ya implementada exitosamente en nuestro centro en los últimos años. En la literatura se ha señalado a la videotoracoscopía como vía de elección para el manejo de los secuestros intralobares y extralobares, incluso en los lactantes menores y recién nacidos ${ }^{21,26}$. También sería un procedimiento de elección para niños mayores en secuestros extralobares complicados ${ }^{13}$ y no complicados $^{27}$. Esta misma técnica se ha descrito para el manejo de los secuestros intralobares en adultos ${ }^{28}$. En nuestra serie se observa que 2 pacientes en que se inició VATS se debió reconvertir, lo cual se ha publicado en otras series ${ }^{26}$.

En el manejo de los secuestros extralobares ectópicos situados en relación a la ubicación hiatal y/o infradiafragmáticos se ha relatado a la laparoscopía como una técnica factible y segura en recién nacidos y lactantes menores ${ }^{29}$.

En este punto se ha indicado a la embolización de la arteria nutricia como una alternativa terapéutica a la cirugía. Chien $^{30}$ describe este procedimiento en 6 pacientes 5 secuestros extralobares y un secuestro intralobar sin apreciarse complicaciones como neumonía recurrente o infecciones en el seguimiento de 13 meses $^{30}$. A pesar de esto, otros autores descartan esta posibilidad dada la dificultad de distinguir entre secuestro y otra patología, el riesgo de infección y las complicaciones propias de la embolización arterial en un estudio retrospectivo en que se analizó embolización versus VATS en 73 pacientes con secuestros pulmonares ${ }^{31}$. Nuevos métodos de embolización han registrado buenos resultados con casuísticas pequeñas ${ }^{32}$.

La evolución de los pacientes en general fue buena, destacando complicaciones como las fístulas broncopleurales ya relatadas previamente, las cuales prolongan la estadía hospitalaria en forma manifiesta. Otras complicaciones descritas como hemotórax, empiemas, fístulas broncopleurocutáneas ${ }^{4}$, no se observaron en el presente estudio.

No registramos mortalidad en nuestra casuística, a diferencia de lo registrado en otras series de casos clínicos ${ }^{4}$.

Las limitaciones de este trabajo son el bajo número de pacientes, el hecho de no haber obtenido de la totalidad de las fichas clínicas las características de la irrigación tanto arterial como venosa, por lo que no se describe el detalle de la irrigación.

Finalmente, se presenta esta serie de casos clínicos que traducen la experiencia de nuestro centro asistencial en los últimos años enfatizándose la importancia de la sospecha diagnóstica adecuada y el manejo multidisciplinario de esta patología.

\section{Bibliografía}

1.- CORBETT H, HUMPHREY G. Pulmonary sequestration. Paediatr Respir Rev 2004; 5: 59-68.

2.- LANGSTON C. New concepts in the pathology of con- 
genital lung malformations. Sem Pediat Surg 2003; 12 : 17-37.

3.- STOCKER J T. The respiratory tract. En: Stocker JT, Dehner LP and Husain AN eds. Pediatric Pathology. Third edition, Philadelphia, Lippincott Williams \& Wilkins. 2011; 441-515.

4.- SAVIC B, BIRTEL F, THOLEN W, FUNKE H, KNOCHE R. Lung sequestration: report of seven cases and review of 540 published cases. Thorax 1979; 34: 96101.

5.- WEI Y, LI F. Pulmonary sequestration: a retrospective analysis of 2625 cases in China. Eur J Cardio-Thorac 2011; 40: e39-e42.

6.- SHEARES B. Recurrent pneumonia in children. Pediatric Annals 2002; 31: 109-114.

7.- HIRAI S, HAMANAKA Y, MITSUI N, UEGAMI S, MATSUURA Y. Surgical treatment of infected intralobar pulmonary sequestration: a collective review of patients older than 50 years reported in the literature. Ann Thorac Cardiovasc Surg 2007; 13: 331-4.

8.- MIRZA B, RAZA A, IJAZ I, IJAZ L, NAZ F, SHEIKH A. Intralobar pulmonary sequestration masquerading as congenital lobar emphysema. Journal of Indian Association of Pediatric Surgeons 2011; 16: 15-7.

9.- CARSIN A, MELY L, CHRESTIAN M A, DEVRED PH, DE LAGAUSIE P, GUYS J M, et al. Association of three different congenital malformations in a same pulmonary lobe in a 5-year-old girl. Pediatric Pulmonology 2010: 45; 832-5.

10.- WOERNER A, SCHWENDENER K, WOLF R, NELLE M. Neonatological and pulmonological management of bilateral pulmonary sequestration in a neonate. World J Pediatr 2008; 4: 301-4.

11.- SHAH R, CARVER T W, RIVARD D C. Torsed pulmonary sequestration presenting as a painful chest mass. Pediatr Radiol 2010; 40: 1434-5.

12.- LIMA M, RANDI B, GARGANO T, TANI G, PESSION A, GREGORI G. Extralobar pulmonary sequestration presenting with torsion and associated hydrothorax. Eur J Pediatr Surg 2010; 20: 208-10.

13.- UCHIDA D A, MOORE K R, WOOD K E, PYSHER T J, DOWNEY E C. Infarction of an extralobar pulmonary sequestration in a young child: diagnosis and excision by video-assisted thoracoscopy. J Laparoendosc Adv Surg Tech A 2010; 20: 399-401.

14.- FILHO D R P, AVINO A J G, BRANDÃO S L B. Extralobar pulmonary sequestration with hemothorax secondary to pulmonary infarction. J Bras Pneumol 2009; 35: 99-102.

15.- VARAS A, BURDACH R, REID A, PAREDES G. Secuestración pulmonar intralobar. Rev Chil Pediatr 1982; 53: 431-41.

16.- KOTECHA S, BARBATO A, BUSH A, CLAUS F, DAVENPORT M, DELACOURT C, et al. Antenatal and postnatal management of congenital cystic adenomatoid malformation. Paediatr Respir Rev 2012; 13: 162-70.
17.- GOMPELMANN D, EBERHARDT R, HEUSSEL CP, HOFFMANN H, DIENEMANN H, SCHUHMANN M, et al. Lung sequestration: a rare cause for pulmonary symptoms in adulthood. Respiration 2011; 82: 445-50.

18.- MONTJOY C, HADIQUE S, GRAEBER G, GHAMANDE S. Intralobar bronchopulmonary sequestra in adults over age 50: case series and review. W V Med J 2012; 108: 8-13.

19.- SEPÚLVEDA W. Perinatal imaging in bronchopulmonary sequestration. J Ultrasound Med 2009; 28: 89-94.

20.- PEDATA R, PALERMO M, MAIELLO M, ESPOSITO N, ERMITO S, DINATALE A, et al. Fetal lung lesions diagnosis: the crucial role of ultrasonography. Journal of Prenatal Medicine 2009; 3: 49-52.

21.- JESCH N K, LEONHARDT J, SUMPELMANN R, GLUER S, NUSTEDE R, URE B M. Thoracoscopic resection of intra- and extralobar pulmonary sequestration in the first 3 months of life. J Pediatr Surg 2005; 40: 1404-6.

22.- LEE E, TRACY D, MAHMOOD S, WELDON C, ZURAKOWSKI D, BOISELLE P. Preoperative MDCT Evaluation of Congenital Lung Anomalies in Children: Comparison of Axial, Multiplanar, and 3D Images. AJR 2011; 196: 1040-6.

23.- BÜYÜKOGLAN H, MAVILI E, TUTAR N, KANBAY A, BILGIN M, OYMAK F, et al. Evaluation of diagnostic accuracy of computed tomography to assess the angioarchitecture of pulmonary sequestration. Tüberküloz ve Toraks Dergisi 2011; 59: 242-7.

24.- SAHINOGLU Z, YUKSEL A, ULUDOGAN M, BILGIC R, TOKSOY G. Left diaphragmatic eventration associated with ipsilateral pulmonary sequestration and intrathoracic kidney in a fetus: reviewing the prenatal diagnosis and etiopathogenesis. Fetal Pediatr Pathol 2011; 30: 233-43.

25.- FERREIRA H P C, FISCHER G B, FELICETTI J C, CAMARGO J J P, ANDRADE C F. Surgical treatment of congenital lung malformations in pediatric patients. J Bras Pneumol 2010; 36: 175-80.

26.- DE LAGAUSIE P, BONNARD A, BERREBI D, PETIT P, DORGERET S, GUYS J M. Video-Assisted thoracoscopic surgery for pulmonary sequestration in children. Ann Thorac Surg 2005; 80: 1266-9.

27.- SUDA T, HASEGAWA S, NEGI K, HATTORI Y. Video-assisted thoracoscopic surgery for extralobar pulmonary sequestration. J Thorac Cardiovasc Surg 2006; 132: 707-8.

28.- GONZÁLEZ D, GARCÍA J, FIEIRA E, PARADELA M. Video-assisted thoracoscopic lobectomy in the treatment of intraloba pulmonary sequestration. Interact Cardiovasc Thorac Surg 2011; 12:77-9.

29.- JOYEUX L, MEJEAN N, ROUSSEAU T, COUAILLIER J F, PIARD F, SAPIN E. Ectopic extralobar pulmonary sequestrations in children: interest of the laparoscopic approach. J Pediatr Surg 2010; 45: 2269-73.

30.- CHIEN K J, HUANG T, LIN CH, LEE CH, HSIEH K, 
WENG K. Early and Late Outcomes of Coil Embolization of Pulmonary Sequestration in Children. Circ J 2009; 73: 938-42.

31.- CHO M J, KIM D Y, KIM S C, KIM K S, KIM E A, LEE B S. Embolization versus surgical resection of pulmonary sequestration: Clinical experiences with a thoracoscopic approach. J Pediatr Surg 2012; 47: 222833.

32.- RODRÍGUEZ L, SUERO P, PRADA F, MORTERA C, MUCHART J, PONS M. Tratamiento endovascular del secuestro pulmonar con dispositivos tipo Amplatzer®. An Pediatr (Barc) 2012; 76: 285-9.

Correspondencia a:

Dr. Guillermo Zepeda F.

Programa de Enfermedades Respiratorias Pediátricas

de la Universidad de Chile.

Magister en Educación en Ciencias de la Salud,

Universidad de Chile.

Fax: 27350445

Email: gezepeda@med.uchile.cl 